International Journal of Modern Physics A

(C) World Scientific Publishing Company

\title{
HADRONIC INTERACTION OF THE $\eta$ MESON WITH TWO NUCLEONS
}

\author{
P. MOSKAL ${ }^{*, \dagger}$, H.-H. ADAM ${ }^{\ddagger}$, A. BUDZANOWSKI ${ }^{\dagger \dagger}$, R. CZYŻYKIEWICZ ${ }^{*, \dagger}$, \\ D. GRZONKA ${ }^{\dagger}$, M. JANUSZ*, L. JARCZYK* , B. KAMYS* , A. KHOUKAZ ${ }^{\ddagger}$, K. KILIAN $^{\dagger}$, \\ P. KLAJA*, J. MAJEWSKI*, ${ }^{*}$, W. OELERT ${ }^{\dagger}$, C. PISKOR-IGNATOWICZ* ${ }^{*}$, J. PRZERWA*, \\ T. ROŻEK ${ }^{\star}, \dagger$, T. SEFZICK ${ }^{\dagger}$, M. SIEMASZKO ${ }^{\star}$, J. SMYRSKI* $^{*}$, A. TÄSCHNER ${ }^{\ddagger}$, \\ P. WINTER ${ }^{\dagger}$, M. WOLKE ${ }^{\dagger}$, P. WÜSTNER ${ }^{\ddagger \ddagger}$, W. ZIPPER ${ }^{\star}$ \\ * Nuclear Physics Department, Jagellonian University, Cracow, 30-059, Poland \\ $\dagger$ Institut für Kernphysik, Forschungszentrum Jülich, Jülich, 52425, Germany \\ ¥ Institut für Kernphysik, Universität Münster, Münster, 48149, Germany \\ ${ }^{\dagger \dagger}$ Institute of Nuclear Physics, Cracow, 31-342, Poland \\ * Institute of Physics, University of Silesia, Katowice, 40-007, Poland \\ $\$ ¥$ ZEL Forschungszentrum Jülich, Jülich, 52425, Germany \\ Received (Day Month Year) \\ Revised (Day Month Year)
}

\begin{abstract}
The COSY-11 collaboration has conducted experiments aiming at the determination of the excitation function and phase-space population of the $p p \rightarrow p p \eta$ reaction close to the kinematical threshold. The precise data obtained with the stochastically cooled proton beam of the cooler synchrotron COSY and the high resolution zero-degree magnetic spectrometer allowed for the observation of the significant deviations - in the shape of the excitation function and two-particle invariant masses - from the predictions based on the assumption that the reaction phase space is homogenously populated. Comparison of the shape of the excitation function for the $p p \rightarrow p p \eta$ and $p p \rightarrow p p \eta^{\prime}$ reaction allows to distinquish in the model independent way an influence originating from the protonproton and proton- $\eta$ interaction. For the comparison the full data set from experiments performed at COSY and other laboratories is used.
\end{abstract}

Keywords: Meson-nucleon interaction, meson production

\section{Introduction}

In analogy to the connection between electromagnetic and Van der Vaals potentials, we may perceive the hadronic force as a residuum of the strong interaction that occurs between quarks and gluons - the constituents of hadrons. Therefore, the knowledge of the interaction of hadrons is interesting not only on its own account but also since it delivers information about the structure of hadrons and the strong interaction itself.

The fact that fourty years after the discovery of the $\eta$ and $\eta^{\prime}$ mesons 1 their interactions with nucleons remain so weakly established, indicates that it is rather challenging to conduct research which could deliver information about this inter- 
action. The scattering length - the very basic quantity describing the low energy interaction potential - in the case of the $\eta$ meson is poorly estimated, and in the case of the $\eta^{\prime}$ meson it is entirely unknown. Estimated value of the real part of the proton- $\eta$ scattering length varies from $0.25 \mathrm{fm}$ to $1.05 \mathrm{fm}$ depending on the approach employed for its determination 2 .

The main obstacle in the experimental studies involving any of neutral ground state mesons is too short life-time which prohibits their utilization as secondary beams. Therefore the study of their interaction with hadrons is accessible only via observations of their influence on the cross section of the reactions in which they were produced (eg. $N N \rightarrow N N$ Meson). The influence of the relatively weak nucleon- $\eta$ interaction may be magnified when producing the meson in the vicinity of two nucleons. In this context the $p p \eta$ system reveals to be particularly interesting since neither the $p p$ nor the $p \eta$ interaction is strong enough to form a bound state and so the $p p \eta$ system may occur to be Borromean. It was pointed out by Wycech 3 that the large enhancement in the excitation function of the $p p \rightarrow p p \eta$ reaction observed close to the kinematical threshold can be described assuming that the proton-proton pair is produced from a large object of a $4 \mathrm{fm}$ radius. Yet, at present it is still not established whether the low energy $p p \eta$ system can really form a Borromean or resonant state. Though the significant progress in the understanding of the production mechanism on the hadronic level has been achieved 451617, the final state system was always treated approximately utterly ignoring the $\eta$-proton final state interaction. Only, very recently the rigorous three-body approach has been applied for the description of the observed excitation function and two-particle invariant mass spectra of the $p p \rightarrow p p \eta$ reaction. Two independent approaches by Deloff 8 and Fix \& Arenhövel 9 revealed that the rigorous three body treatment of the final state leads to the results significantly different from the two-body approach even when the $\eta$-proton interaction is completely disregarded. Also a very important conclusion drawn in reference 9 is that the role of the final state interaction depends crucially on the range of the primary reaction dynamics, showing that the inaccuracy of establishing contributions from the exchange of various mesons limits the inferences about the interaction among the final state particles.

The data which stimulated the development of the abovementioned three body formalism will be presented hereafter. For the better visualisation of the effects due to the proton- $\eta$ interaction we will compare the shape of the excitation functions of the $p p \rightarrow p p \eta$ and $p p \rightarrow p p \eta^{\prime}$ reactions and will discuss the observed differences. In the next section which constitutes an extraction from the more comprehensive treatise 10 we will present only the excitation function and for the details concerning the differential distributions the reader is referred to the more exhaustive experimental 101112 and theoretical elaborations 5191314 . 


\section{Excitation function of the reactions $p p \rightarrow p p \eta$ and $p p \rightarrow p p \eta^{\prime}$}

The determined energy dependences of the total cross section for $\eta^{\prime} 1516$ and $\eta 111617$ mesons production in proton-proton collisions are presented in figure 1 Comparing the data to the arbitrarily normalized phase space integrals (dashed lines) reveals that the $p p \eta$ FSI enhances the total cross section by more than an order of magnitude for low excess energies.

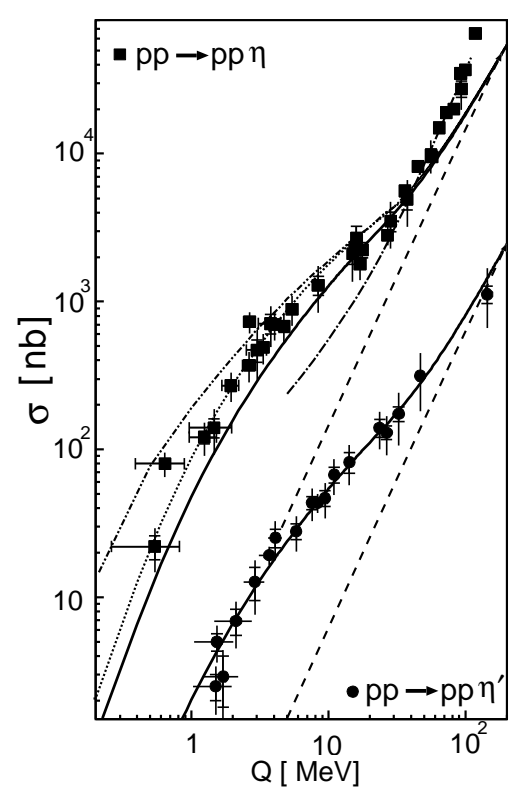

Fig. 1. Total cross section for the reactions $p p \rightarrow$ $p p \eta^{\prime}$ (circles) and $p p \rightarrow p p \eta$ (squares) as a function of the centre-of-mass excess energy Q. Data are from refs. 11151617 The dashed lines indicate a phase space integral normalized arbitrarily. The solid lines show the phase space distribution with inclusion of the ${ }^{1} S_{0}$ proton-proton strong and Coulomb interactions. In case of the $p p \rightarrow p p \eta$ reaction the solid line was fitted to the data in the excess energy range between 15 and $40 \mathrm{MeV}$. Additional inclusion of the proton- $\eta$ interaction is indicated by the dotted line. The scattering length of $a_{p \eta}=0.7 \mathrm{fm}+i 0.4 \mathrm{fm}$ and the effective range parameter $b_{p \eta}=-1.50 \mathrm{fm}-i 0.24 \mathrm{fm}{ }^{18}$ have been chosen arbitrarily. The dashed-dotted line represents the energy dependence taking into account the contribution from the ${ }^{3} P_{0} \rightarrow{ }^{1} S_{0} s,{ }^{1} S_{0} \rightarrow{ }^{3} P_{0} s$ and ${ }^{1} D_{2} \rightarrow{ }^{3} P_{2} s$ transitions 13. Preliminary results for the ${ }^{3} P_{0} \rightarrow{ }^{1} S_{0} s$ transition with full treatment of three-body effects are shown as a dashed-double-dotted line 9 . The absolute scale of dashed-double-dotted line was arbitrary fitted to demonstrate the energy dependence only.

One recognizes also that in the case of the $\eta^{\prime}$ meson the data are described very well (solid line) assuming that the on-shell proton-proton amplitude exclusively determines the phase space population. In the case of $\eta$ meson production the interaction between nucleons is evidently not sufficient to describe the increase of the total cross section for very low and very high excess energies, as can be concluded from the comparison of the data and the upper solid line in figure 1 This line was normalized to the data at an excess energy range between $15 \mathrm{MeV}$ and $40 \mathrm{MeV}$. The enhancement of the total cross section for higher energies can be assigned to the outset of higher partial waves, and the discrepancy visible closer to the threshold can be plausibly explained by the influence of the attractive interaction between the $\eta$ meson and the proton. A similar effect close-to-threshold is also observed in the data of photoproduction of $\eta$ via the $\gamma d \rightarrow p n \eta$ reaction 19 indicating to some extent that the phenomenon is independent of the production process and rather is related to the interaction among the $\eta$ meson and nucleons.

The dotted-line in figure 1 corresponds to the simple phenomenological treatment 20 based on the factorization of the transition amplitude into the constant primary production and the on-shell incoherent pairwise interaction among outgoing particles. Although it describes the enhancement close-to-threshold very well, 
it fails to describe the invariant mass distribution of the proton-proton and proton$\eta$ subsystems determined recently at $\mathrm{Q}=15 \mathrm{MeV}$ by the COSY-TOF 12 and at $\mathrm{Q}=15.5 \mathrm{MeV}$ by the COSY-11 11 collaborations. It was suggested in reference 13 that the bump in the invariant mass spectra may be due to the contribution from higher partial waves. However, the amount of the P-wave admixture derived from the proton-proton invariant mass distribution spoils significantly the agreement with the data at low values of $\mathrm{Q}$ (see dashed-dotted line in figure 11). Another explanation proposed is based on the anticipation that the production amplitude may vary with energy 14. However, both listed approaches neglected the proton- $\eta$ interaction and only now the first calculations with the rigorous treatment of the three-body final state including both proton-proton and proton- $\eta$ interaction are available. And although biased with neglection of the Coulomb effect 9 or imaginary part of the proton- $\eta$ scattering length $[8$ they herald a fully rigorous explanation of the observed spectra.

\section{Acknowledgments}

The work has been supported by the DAAD Exchange Programme (PPPPolen) and by the Polish State Committee for Scientific Research (grant No. PB1060/P03/2004/26).

\section{References}

1. A. Pevsner et al., Phys. Rev. Lett. 7 (1961) 421; G. R. Kalbfleisch et al., Phys. Rev. Lett. 12 (1964) 527; M. Goldberg et al., Phys. Rev. Lett. 12 (1964) 546.

2. A. M. Green, S. Wycech, e-Print Archive: nucl-th/0411024

3. S. Wycech, Acta Phys. Pol. B 27 (1996) 2981.

4. G. Fäldt, T. Johansson, C. Wilkin, Physica Scripta T 99 (2002) 146.

5. V. Baru et al., Phys. Rev. C 67 (2003) 024002.

6. K. Nakayama, H. Haberzettl, Phys. Rev. C 69 (2004) 065212.

7. V. Bernard, N. Kaiser, Ulf-G. Meissner, Eur. Phys. J. A 4 (1999) 259.

8. A. Deloff, e-Print Archive: nucl-th/0406069

9. A. Fix, H. Arenhövel, Phys. Rev. C 69 (2004) 014001.

10. P. Moskal, e-Print Archive: hep-ph/0408162

11. P. Moskal et al., Phys. Rev. C 69 (2004) 025203.

12. M. Abdel-Bary et al., Eur. Phys. J. A 16 (2003) 127.

13. K. Nakayama et al., Phys. Rev. C 68 (2003) 045201.

14. A. Deloff, Phys. Rev. C 69 (2004) 035206.

15. F. Balestra et al., Phys. Lett. B 491 (2000) 29; R. Wurzinger et al., Phys. Lett. B 374 (1996) 283; P. Moskal et al., Phys. Lett. B 474 (2000) 416; A. Khoukaz et al., Eur. Phys. J. A 20 (2004) 345; P. Moskal et al., Phys. Rev. Lett. 80 (1998) 3202.

16. F. Hibou et al., Phys. Lett. B 438 (1998) 41.

17. A. M. Bergdolt et al., Phys. Rev. D 48 (1993) R2969; E. Chiavassa et al., Phys. Lett. B 322 (1994) 270; H. Calén et al., Phys. Lett. B 366 (1996) 39; H. Calén et al., Phys. Rev. Lett. 79 (1997) 2642; J. Smyrski et al., Phys. Lett. B 474 (2000) 182.

18. A. M. Green, S. Wycech, Phys. Rev. C 55 (1997) R2167.

19. V. Hejny et al., Eur. Phys. J. A 13 (2002) 493; Ch. Elster et al., e-Print Archive: nucl-th/0207052 A. Sibirtsev et al., Phys. Rev. C 65 (2002) 067002.

20. P. Moskal, M. Wolke, A. Khoukaz, W. Oelert, Prog. Part. Nucl. Phys. 49 (2002) 1. 